\title{
RESEARCH HIGHLIGHT Ubiquitin-induced phase separation of p62/SQSTM1
}

\author{
Lina Herhaus ${ }^{1}$ and Ivan Dikic ${ }^{1,2}$ \\ Cell Research (2018) 28:389-390; https://doi.org/10.1038/s41422-018-0030-x
}

\begin{abstract}
During the process of autophagy, cytoplasmic material is targeted by autophagic receptor proteins, which were thought to segregate the cargo by forming aggregates. Recent evidence suggests that cargo is actually concentrated and segregated into protein condensates by ubiquitin chaininduced phase separation of autophagy receptor p62.
\end{abstract}

Autophagy is vital for cellular homeostasis, as damaged proteins or organelles are engulfed, and degraded, and nutrients are recycled. ${ }^{1}$ Autophagosomal membranes form and expand at the site of cytosolic disposal and are critical for separation and delivery of cargo to the lysosome. Autophagosomes are characterized by double lipid membranes conjugated with the Atg8 family of ubiquitin-like modifiers. There are six members of autophagy modifiers in mammalian cells (named LC3s and GABARAPs) that can bind to a variety of autophagy receptor or adaptor proteins through their LIR (LC3-interacting region) motif., ${ }^{2,3}$ One such autophagy receptor protein is p62 or SQSTM1. p62 is a multimodular protein, which harbors a LIR motif, a PB1 (Phox and Bem1) domain used for oligomerization and a UBA (ubiquitinassociated) domain to bind ubiquitin chains. Through these various domains, p62 links and recruits ubiquitylated cytosolic cargo material to the autophagic isolation membrane. ${ }^{4,5}$ The oligomerization of p62 through the PB1 domain generates higher avidity interactions with ubiquitin and LC3-coated surfaces. ${ }^{6}$ p62 preferentially binds to K63-linked ubiquitin chains, which are generated by the action of E1, E2 and E3 enzymes that link a ubiquitin molecule on lysine 63 to the $\mathrm{N}$-terminus of the next ubiquitin molecule. Proteins destined for degradation are usually decorated with ubiquitin chains, which are recognized by the proteasome or autophagy receptor proteins, e.g., p62 binds to the ubiquitylated cargo proteins and forms protein aggregates, which are then degraded by autophagy. Upon autophagic malfunctions or p62 mutations, p62 protein aggregates are a common component of cellular inclusion bodies found in several diseases affecting the brain and liver. ${ }^{7}$

Recent findings by Sun et al. show that the p62 cellular aggregates can undergo fusion and resemble liquid-like, nonmembrane-bound compartments. ${ }^{8}$ Therefore, the observed p62 accumulations could be considered biomolecular condensates rather than aggregates. These are membrane-less, micron-scale compartments, which behave as phase-separated liquids that concentrate proteins or nucleic acids through multivalent macromolecular interactions. ${ }^{9}$ In vivo p62 forms spherical liquid-like droplets that can undergo fusion, however, this phase separation only occurs in the presence of ubiquitin chains bound by $\mathrm{p} 62$ polymers, thereby driving autophagic cargo concentration and segregation (Fig. 1). ${ }^{8}$
In cells, p62 exists in two populations: the membrane-bound form that interacts with autophagosomes and the cytosolic pool. To decipher the precise nature of observed cellular membrane-less p62 accumulations, Sun et al. made use of autophagy-deficient $A T G 12^{-1-}$ cells. $^{8}$ In these cells p62 cannot be taken up by autophagosomes and the membrane-less accumulated p62 is observed as viscous liquid droplets arising from phase separation. p62 present in these droplets can exchange with other p62-containing droplets as shown by recovery after photobleaching.

Next, the authors sought to investigate the organization of p62mediated biomolecular condensate formation. Phase separation is not induced by $\mathrm{p} 62$ polymer formation alone or by the presence of monoubiquitin. In addition to p62, other polyubiquitylated cargo proteins or free ubiquitin chains need to be present to induce segregation into droplets. The nature of these ubiquitin chains should preferentially be K63- or M1-linked chains with at least 8 ubiquitin molecules (although K48-linked polyubiquitylated cargo can also induce phase separation). While p62 has been shown to tightly bind ubiquitin chains, they diffuse separately through the droplets, as determined by different recovery rates after photobleaching, indicating that they do not form a stable complex. Likewise, the autophagy modifier LC3 is recruited into these bodies, however, it recovers quicker than p62 from photobleaching, indicating its separate diffusion from the p62induced biomolecular condensates. ${ }^{8}$

For phase separation and subsequent autophagic degradation in vivo, p62 needs to form polymers (through its PB1 domain) and bind ubiquitin via the UBA domain. Many post-translational modifications have been identified within the UBA domain of p62. During autophagic induction, the UBA domain of p62 is phosphorylated on S403 by CK2 or TBK1, which increases p62binding affinity towards ubiquitin chains in cells, ${ }^{10,11}$ thus also promoting polyubiquitin chain-induced p62-mediated phase separation and autophagic degradation. ${ }^{8}$ Mutations in the UBA domain of p62 (M404T and G411S) have been identified as the cause of Paget's disease of bone. ${ }^{12}$ Sun et al. were able to show that both of these mutations negatively affected ubiquitin chaininduced p62 phase separation and autophagic degradation. ${ }^{8}$

Non-membrane-bound Atg8 proteins were also detected within p62 droplets. $^{8}$ Since LC3s and GABARAPs can bind many LIR motifcontaining proteins, the in vivo protein composition of these biomolecular condensates could be much more diverse than expected. It would be interesting to isolate the p62-containing condensates, extract ubiquitylated cargo proteins and then analyze the total protein composition by mass spectrometry. The recruitment of distinct LIR motif-containing proteins during

\footnotetext{
${ }^{1}$ Institute of Biochemistry II, Goethe University School of Medicine, Theodor-Stern-Kai 7, Frankfurt am Main 60590, Germany and ${ }^{2}$ Buchmann Institute for Molecular Life Sciences, Goethe University Frankfurt, Riedberg Campus, Max-von-Laue-Straße 15, Frankfurt am Main 60438, Germany

Correspondence: Ivan Dikic (ivan.dikic@biochem2.de)
}

Published online: 23 March 2018 


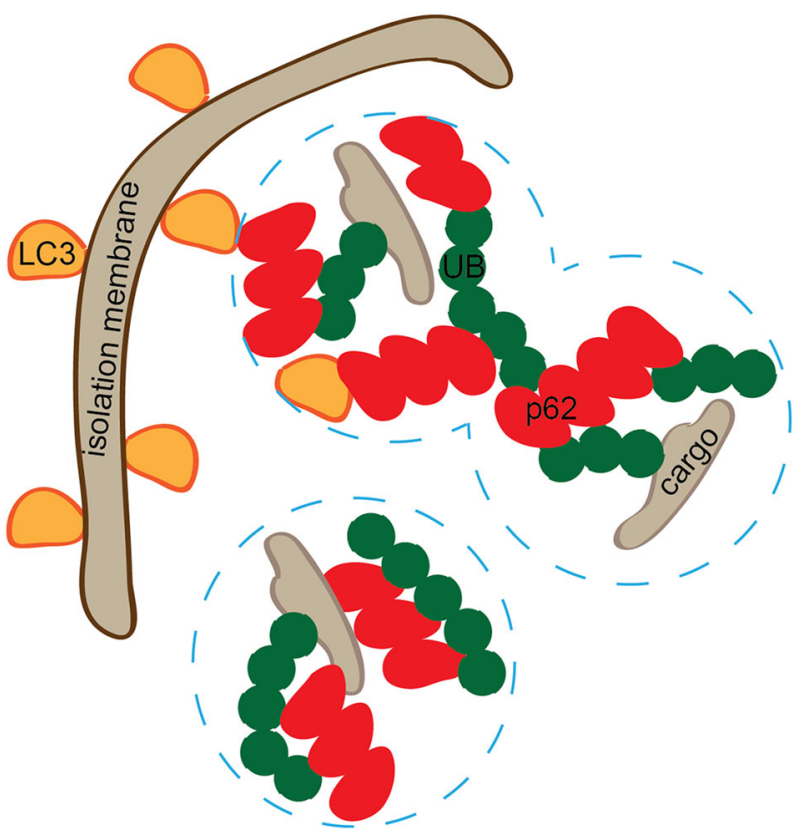

Fig. 1 Cartoon of p62-mediated biomolecular condensate formation. p62 can be membrane-bound or free in the cytosol, where it forms polymers and induces phase separation together with ubiquitin chains. Phase separation results in the formation of liquid-like droplets, which are spherical and can undergo fusion. LC3 family proteins can also be present within these droplets and upon binding to p62 and LC3 lipidation to autophagosomes, the biomolecular condensates can be degraded through autophagy. LC3 family proteins are depicted in orange, p62 polymers in red, ubiquitin chains in green, cargo proteins and the isolation membrane in brown, and biomolecular condensates in blue

different autophagy-induced cellular stresses could give more insights into autophagy signaling and selectivity.
In summary, these recent findings alter the view of our current understanding of p62-mediated autophagic degradation, in which cargo proteins are concentrated and segregated into rigid p62containing aggregates. Ubiquitin and p62-mediated biomolecular condensate formation allow proteins present in these droplets to diffuse freely, retain their conformation and thus might allow for biochemical reactions to take place. ${ }^{8}$ Whether or how the p62induced biomolecular condensates can act as organizing platforms for signaling cascades that modulate autophagy requires further investigation.

Biomolecular condensates are involved in diverse cellular processes, such as ribosome biogenesis and RNA metabolism. Liquid-liquid phase separation appears to be a common mechanism for the generation of diverse biomolecular condensates and phase separation is induced through multivalent macromolecular interactions of different interacting proteins. ${ }^{9}$ The composition and assembly of these membrane-less compartments is mostly known, however, to which extent these biomolecular condensates act as signaling platforms remains to be fully understood.

\section{REFERENCES}

1. Lamb, C. A., Yoshimori, T. \& Tooze, S. A. Nat. Rev. Mol. Cell. Biol. 14, 759-774 (2013).

2. Stolz, A., Ernst, A. \& Dikic, I. Nat. Cell Biol. 16, 495-501 (2014).

3. Khaminets, A., Behl, C. \& Dikic, I. Trends Cell. Biol. 26, 6-16 (2016).

4. Lamark, T., Svenning, S. \& Johansen, T. Essays Biochem. 61, 609-624 (2017).

5. Katsuragi, Y., Ichimura, Y. \& Komatsu, M. FEBS J. 282, 4672-4678 (2015).

6. Wurzer, B. et al. elife 4, e08941, (2015).

7. Yamamoto, A. \& Simonsen, A. Neurobiol. Dis. 43, 17-28 (2011).

8. Sun, D., Wu, R., Zheng, J., Li, P. \& Yu, L. Cell Res. https://doi.org/10.1038/s41422018-0017-7 (2018).

9. Banani, S. F., Lee, H. O., Hyman, A. A. \& Rosen, M. K. Nat. Rev. Mol. Cell. Biol. 18, 285-298 (2017).

10. Matsumoto, G., Wada, K., Okuno, M., Kurosawa, M. \& Nukina, N. Mol. Cell 44, 279-289 (2011).

11. Richter, B. et al. Proc. Natl Acad. Sci. USA 113, 4039-4044 (2016).

12. Rea, S. L., Walsh, J. P., Layfield, R., Ratajczak, T. \& Xu, J. Endocr. Rev. 34, 501-524 (2013). 